\section{A SEARCH FOR THE "HOLY GRAIL" IN THE EVALUATION OF FEBRILE NEONATES AGED 28 DAYS OR LESS: A PROSPECTIVE STUDY}

\author{
E. Bilavsky, L.A. Hoffnung, H.Y. Bilavsky, J. Amir, \\ G. Livni
}

Schneider Medical Center, Petah-Tikva, Israel

Objective: To prospectively determine the reliability of low-risk criteria to exclude serious bacterial infection (SBI) in febrile neonates aged $\leq 28$ days.

Patients and methods: All febrile neonates aged $\leq 28$ days who were hospitalized for fever evaluation from 2005 to 2009 were prospectively divided into two groups by risk status for SBI. The following criteria were used to define low risk:

(1) unremarkable medical history;

(2) well-appearing;

(3) no focal signs of infection;

(4) white blood cell count between 5000 to $15000 /$ $\mathrm{mm}^{3}$;

(5) normal urinalysis;

(6) no mucoid or bloody diarrhea.

Findings were analyzed against the culture results, and routine validity measures and positive and negative likelihood ratios were calculated.

Results: Of the 465 neonates who met the inclusion criteria, 177 (38.1\%) were considered at high risk for SBI and 288 (61.9\%) low risk. On culture, SBIs were found in 55 (31.1\%) neonates in the high-risk group compared to $10(3.5 \%)$ in the low-risk group $(p<0.001)$. The sensitivity, specificity, positive predictive value and negative predictive value of the criteria for all types of SBI were $84.6 \%(95 \%$ $\mathrm{Cl}-73.9 \%-91.4 \%)$, 69.5\% (95\% Cl 64.8\%-73.8\%), $31 \%(95 \% \mathrm{Cl}-27.3 \%-35.1 \%)$ and $96.5 \%(95 \% \mathrm{Cl}-$ $94.3 \%-98 \%)$, respectively.

Conclusion: The defined criteria are a useful tool for predicting SBI in neonates. However, they are not sufficiently reliable to exclude an SBI or an invasive SBI. It is therefore suggested that all febrile neonates in this age group should be hospitalized for complete evaluation and consideration of empirical intravenous antibiotic treatment.

\section{RECURRENT WHEEZING IN THIRD YEAR AMONG CHILDREN BORN AT 32 WEEKS GESTATION: RELATIONSHIP RESPIRATORY SYNCYTIAL VIRUS DURING FIRST YEAR}

G. Escobar ${ }^{1}$, A. Ragins ${ }^{1}$, S.X. Li ${ }^{1}$, L. Prager ${ }^{2}$, A.S. Masaquel ${ }^{3}$, P. Kipnis ${ }^{1}$

${ }^{1}$ Systems Research Initiative and Perinatal Research Unit, Kaiser Permanente Division of Research, Oakland, '2Department of Pediatrics, Kaiser Permanente Medical Center, Daly City, CA,

${ }^{3}$ MedImmune, LLC, Gaithersburg, MD, USA

Background: Respiratory syncytial virus (RSV) is responsiblefor $50-80 \%$ of bronchiolitis hospitalizations and the leading cause of hospitalization in children < 1 year.

Objective: To quantify the relationship between recurrent wheezing (RW) in year three and documented RSV infection, prematurity, and neonatal oxygen exposure.

Design/methods: Participants were 71,102 children born at $\geq 32$ weeks gestational age (GA)who were members of an integrated health care delivery system in Northern California for 9 months in their first and third years. The principal exposures were laboratoryconfirmed, medically-attended RSV infection during year one, and receipt of supplemental oxygen during birth hospitalization. The outcome was RW in year three.

Results: Rate of RW was $16.23 \%$ among premature infants with RSV infection and $6.22 \%$ among those without. Infants having an RSV outpatient encounter (adjusted odds ratio [AOR] 2.07, 95\% Cl, 1.61-2.67), uncomplicated hospitalization (AOR 4.66, 95\% Cl, 3.55-6.12), or prolonged hospitalization (AOR 3.42, $95 \% \mathrm{Cl}, 2.01-5.82$ ) were at increased risk for RW compared to infants without RSV infection. GA of 3436 weeks was associated with increased risk for RW (AOR 1.23, 95\% Cl 1.071.41) compared to 38-40 weeks, while GA of 41 weeks was protective (AOR $0.90,95 \% \mathrm{Cl}, 0.81-0.99)$. Supplemental oxygen exposure was associated with increased risk at all levels. The 3 most important predictors were infant sex, family's asthma history, and RSV infection.

Conclusions: Laboratory-confirmed, medicallyattended RSV infection, prematurity, and exposure to supplemental oxygen during the neonatal period have independent associations with the development of third year RW.

The study was sponsored by Medlmmune. 